\title{
Controlled processing in pigeons
}

\author{
RUTH M. COLWILL \\ University of Cambridge, Cambridge, England
}

\begin{abstract}
This paper investigates whether rehearsal, or posttrial processing, of information about an event can be modulated by instructions in infrahuman organisms. Using pigeons, a procedure was developed for establishing a stimulus as a cue to disengage rehearsal, or posttrial processing, of a food reinforcer. Specifically, the stimulus instructed the animals to behave on a subsequent choice test as if food had not, in fact, been presented. Results are reported that suggest that this training was successful in establishing the stimulus as a cue to forget the occurrence of the food. These findings are interpreted as providing tentative support for the view that the cognitive repertoire of infrahuman organisms does include controlled processing strategies.
\end{abstract}

Modern theories of information processing typically draw a distinction between automatic processing that proceeds inflexibly and involuntarily and controlled processing that is instigated voluntarily and regulated by the subject (Hasher \& Zacks, 1979; LaBerge \& Samuels, 1974; Posner \& Snyder, 1975; Shiffrin \& Schneider, 1977). Although automatic processes seem not to be involved in learning (Shiffrin \& Schneider, 1977; but see Hasher \& Zacks, 1979), controlled processing is assumed to play an integral part in the acquisition of new information. Several studies using human subjects have reported that an explicit instruction to rehearse information enhances learning about that information (Bower \& Winzenz, 1970; Craik \& Tulving, 1975; Glenberg, Smith, \& Green, 1977; Rundus, 1971). Other studies have shown that instructions to forget the target information reduce learning, as evidenced by poor performance on recall tests (Bjork, 1972). One interpretation of these results is that the subject uses the instruction as a cue to implement or terminate the appropriate processing strategies.

Recently, a number of attempts have been made to develop procedures for investigating controlled processing in animals (cf. Grant, 1981a; Maki, 1981). Usually, subjects are presented with an item that is followed by one of two cues. Retention of the item is always tested after one cue (cue to remember) but never after the other cue (cue to forget). Following this training, probe trials are administered in which retention is tested for the item regardless of the identity of the cue. In some cases, retention of an item is made worse by the presentation of a cue to forget (Grant, 1981b; Kendrick, Rilling, \& Stonebraker, 1981; Stonebraker \& Rilling, 1981), but in other

This work was supported by a postgraduate research grant from the Medical Research Council and a grant from the Science Research Council. I should like to thank Anthony Dickinson for invaluable suggestions regarding the execution of this work and preparation of the manuscript. I am also grateful to Robert Rescorla and Bill Whitlow for many helpful comments.

The author's present mailing address is: The Psychological Laboratory, University of Cambridge, Downing Street, Cambridge CB2 3EB, England. cases, it is unaffected (Kendrick et al., 1981). A number of explanations have been offered for these different outcomes (Grant, 1982; Maki, 1981; Stonebraker, Rilling, \& Kendrick, 1981). However, none of the procedures used in these studies explicitly demands that subjects modify posttrial processing. In the experiments with humans in which multiple events are presented on any one trial, it is clearly beneficial for subjects to suppress rehearsal of forget cued information so that more processing time can be allocated to the remember cued information. Such an advantage is not afforded to animals in a procedure in which a single item is presented on any one trial. Consequently, the failure to observe a systematic decrement in performance on probe retention trials may be due to ineffective training procedures: simply making a cue to forget signal a trial outcome that is irrelevant to the preceding event may be insufficient to encourage the subject to adopt an active strategy for suppressing rehearsal of information about that event.

This paper describes an alternative technique for studying controlled processing that permits a distinct advantage to be gained from modifying posttrial processing of a target event. For this purpose, the present experiment exploited an asymmetry in the performance of pigeons on a delayed conditional discrimination (DCD) in which food served as a sample stimulus. In that procedure, subjects were reinforced for selecting one of two choice stimuli if their presentations had been shortly preceded by the delivery of food. Selection of the other choice stimulus was reinforced if no sample had been presented on that trial. It has been repeatedly observed that the probability of making the correct choice response on food sample trials declines systematically as the interval between the presentation of the food sample and the opportunity to make the choice response is increased (Colwill, 1982; Colwill \& Dickinson, 1980). Performance on no-sample trials, however, is unaffected by the length of the retention interval and remains stable and accurate across delays of up to $1 \mathrm{~h}$. This asymmetry in performance on foodsample and no-sample trials has been interpreted as indicating that different processes control choice respond- 
ing on the two types of trials. Only performance on foodsample trials is assumed to be governed by a memory trace. In the absence of a memory trace for that event, the choice response appropriate for a no-sample trial is made. From this, it follows that if a cue were presented after the food sample that indicated that the correct response was the one appropriate to a no-sample trial, it would be of considerable advantage to the subject to forget the occurrence of food: by doing so, the memory of food would be less able to interfere with selection of the correct choice stimulus. Thus, the cue might well develop the capacity to cancel the memory of the food sample.

The purpose of this experiment was to investigate whether controlled processing strategies were in fact part of the cognitive repertoire of infrahuman subjects. To this end, a group of pigeons were trained on two concurrent DCDs with food-sample and no-sample trials. On half of each type of trial, two different colors, red and green, were designated as the choice stimuli, whereas on the remaining trials two gratings differing in line orientation, horizontal and vertical, served as the choice stimuli. Choice of one color and one orientation was reinforced on food-sample trials; otherwise choice of the other member of the pair was reinforced. When a reasonable level of performance had been obtained on trials with a 3-sec retention interval, all subjects were shifted to a training regimen designed to establish an auditory stimulus as a signal to forget the occurrence of an immediately preceding food sample. This was accomplished by scheduling additional DCD trials (directed-forgetting trials) that were always initiated by a food sample that was followed by the presentation of a clicker for $3 \mathrm{sec}$. This always terminated with the presentation of the red and green choice stimuli. Choice of the color that had previously been reinforced on food-sample trials was not reinforced on these directed forgetting trials. Instead, choice of the color appropriate to a no-sample trial was reinforced.

There are two ways in which the pigeons could learn to solve this task. They could learn to use the forget cue as a signal not to engage in posttrial processing of the food sample and to respond as if no sample had been presented. Alternatively, they could simply treat the task as a complex conditional discrimination problem. Thus, they might learn to respond to one choice stimulus on the basis of information about the clicker or food-clicker compound in short-term memory and to the other choice stimulus if a memory trace for food alone existed in short-term memory. In order to assess which of these two strategies had been used, transfer trials were administered that were identical to the directed forgetting trials except that the two orientation stimuli rather than the two colors were presented as the choice stimuli. It was anticipated that if directed forgetting training had been successful in endowing the clicker with the capacity to cancel the memory of the food sample, the birds should reject the choice key that was correct on food-sample trials and should tend to choose the orientation appropriate for a no-sample trial. However, if the problem had been solved as a conditional discrimination, there would be no information relevant to guide choice responding on these transfer trials. This could lead to one of two outcomes. First, if the cue to forget were simply ignored and choice responding was based on the trace of the food sample in short-term memory, the choice key appropriate to a food sample trial should be selected. Alternatively, if the cue to forget had any general interference effects, choice of the orientation appropriate to food should be disrupted and performance could be reduced to chance level. On the basis of this account, there seemed to be no reason to expect that selection of the orientation appropriate to the food sample would fall below chance.

\section{METHOD}

\section{Subjects}

Nineteen pigeons, which had been used in a study exploring the effect of ambient illumination on performance in food-sample and nosample DCD trials with red and green choice stimuli, served as subjects. They were maintained at $80 \%$ of their free-feeding weights by food deprivation and were housed in individual cages with water freely available.

\begin{abstract}
Apparatus
Training and testing were conducted in two Campden Instruments pigeon chambers enclosed in dark, sound-attenuating chests. A standard Campden Instruments intelligence panel with an additional key inserted midway between and level with the other keys constituted one wall of each chamber. The side keys could be transilluminated with red and green light and could display gratings of horizontal and vertical white bars. The chambers could be dimly illuminated by a houselight located directly above the center key on the intelligence panel. An aperture giving access to the food magazine was situated below the center key and was illuminated with white light whenever food was presented. Each chamber was equipped with a loudspeaker mounted on the left-hand side of the magazine aperture. This was used for the presentation of a 20-Hz clicker generated by a Campden Instruments Audio generator. Each chamber was interfaced with an Acorn microprocessor which controlled the stimulus events and monitored responses.
\end{abstract}

\section{Procedure}

Autoshaping. All subjects were given two sessions of autoshaping, consisting of $\mathbf{4 0}$ trials with an intertrial interval (ITI) of $20 \mathrm{sec}$. A trial began with the presentation of a horizontal or vertical grating stimulus on either the right or left key. After $8 \mathrm{sec}$, the key darkened and food was presented for $3 \mathrm{sec}$. The houselight remained on throughout illumination of the side key and during the ITI. The frequency of a particular stimulus on each side key was counterbalanced within a session.

Delayed conditional discrimination (DCD) training. The subjects initially received 10 DCD sessions, each of which consisted of 48 trials presented in a semirandom order. Half the trials began with the presentation of a 3-sec food sample, which was followed, after a dark variable retention interval, by the illumination of the side keys with the horizontal and vertical choice stimuli. On the remaining trials, no sample was presented and the houselight was simply extinguished. After a period of time comparable to that of a corresponding food sample trial, the choice stimuli were presented. On both food-sample and no-sample trials, a single peck on either key darkened both. Choice of one orientation was reinforced if the trial had started with a food-sample presentation, otherwise choice of the other orientation was reinforced. Incorrect choices initiated a 3-sec period of darkness.

The retention interval was 0 sec on all trials for the first six sessions. For half the trials in the following four sessions it remained at $0 \mathrm{sec}$, but on the remaining trials it was increased to $3 \mathrm{sec}$. Choice of the vertical grating was designated correct on food-sample trials for 11 sub- 
jects. For the remaining 8 animals, choice of the horizontal grating was reinforced on food-sample trials.

A further 10 DCD sessions, each containing 48 trials, were then administered to reestablish DCD performance with the red and green choice stimuli and to continue training with the orientation choice stimuli. This was accomplished by presenting the color choice stimuli on half of the food sample and no-sample trials and the orientations on the remainder of the trials. The reinforcement contingencies operating on trials with the color choice stimuli were consonant with those experienced by these subjects in their previous study. The retention interval was $3 \mathrm{sec}$ on all trials, which were presented in a semirandom sequence. Throughout this stage of training, the ITI was $30 \mathrm{sec}$.

Directed-forgetting training. All subjects received 44 daily training sessions, each containing $\mathbf{4 0}$ trials. Thirty-two of these trials were identical to those programmed in the final phase of DCD training. There were equal numbers of food and no-sample trials with colors and orientations serving as choice stimuli. Choice responding was reinforced in accordance with past contingencies. The remaining 8 trials constituted directed-forgetting trials. These were similar to the food-sample trials with colors as choice stimuli except that a clicker was presented throughout the 3 -sec retention interval. In addition, the reinforcement contingencies differed. On these trials, choice of the color previously reinforced on no-sample trials was reinforced.

The trials were scheduled in a quasi-random order such that within a block of 10 trials there was an even distribution of trial types counterbalanced with respect to the configuration of the choice stimuli on the side keys. The houselight was illuminated throughout the 30 -sec ITI.

The directed forgetting task proved to be very difficult and 11 of the 19 subjects failed to solve it despite extensive training. Following introduction of the directed forgetting trials, the pigeons' behavior broke down completely on food-sample trials with the colored choice stimuli and they consistently chose the color appropriate for the no-sample trial.
Consequently, these animals were excluded from the experiment and their data were discarded.

Testing. Two transfer test sessions were administered to the eight remaining subjects in an attempt to determine whether or not the clicker was functioning as an instruction to terminate posttrial processing. These sessions were identical to the directed-forgetting training sessions except that four transfer test trials were included. These trials were initiated by the food sample, which was followed by a 3-sec retention interval throughout which the clicker was presented. This terminated with the presentation of the orientation choice stimuli. A single peck on either side key darkened both and initiated the ITI. Food reinforcement for correct responses was withheld on these trials to ensure that selection of a particular choice key mirror past reinforcement contingencies rather than reflect the impact of new ones.

\section{RESULTS}

Figure 1 shows $D C D$ performance during the $10 \mathrm{DCD}$ training sessions that preceded directed forgetting training and during the 44 directed-forgetting training sessions. DCD performance is shown separately on food-sample and no-sample trials with colors and orientations as choice stimuli and is presented in two-session blocks. Performance on the directed-forgetting trials (trials on which the food sample was followed by the clicker), is not plotted on this graph. Following the introduction of directedforgetting trials, Session 11 onwards, performance on food-sample trials with colored choice stimuli declined initially but then recovered towards the end of training

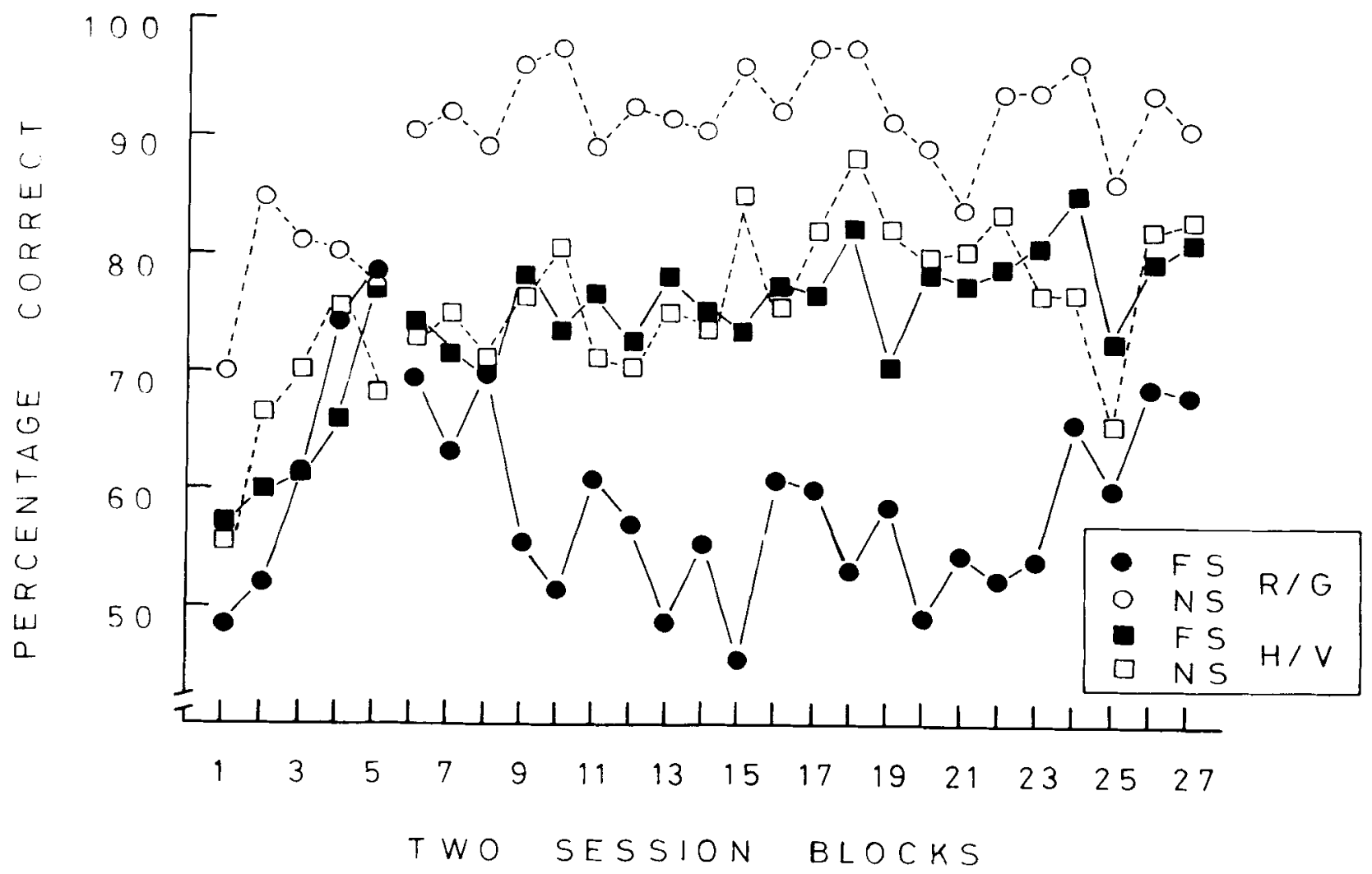

Figure 1. Performance on food-sample (FS, solid symbols) and no-sample (NS, open symbols) DCD trials with colored (R/G, circles) and line orientation (H/V, squares) choice stimuli during the final 10 sessions of DCD training and throughout the directedforgetting training. 
to a level slightly below that attained on food-sample and no-sample trials with orientation choice stimuli. Performance on these trials was stable and nondifferential. Choice responding was most accurate on no-sample trials with colored choice stimuli. An analysis of these data for the last two-session block revealed a significant interaction between type of sample and pair of choice stimuli $[F(1,7)=7.28, p<.05]$. Subsequent analyses indicated that performance was better on no-sample trials than on food sample trials with colored choice stimuli $[\mathrm{F}(1,7)=$ $8.79, p<.05$ ], but equivalent on trials with orientations $(\mathrm{F}<1)$.

For clarity of exposition, performance on trials is illustrated in Figure 2 together with that of performance on control-food sample trials with the colored choice stimuli as a function of the percentage choice of the color approriate to the food sample. Initially, the probability of choosing the choice key corresponding to the food sample was high on directed-forgetting trials. However, with continued training, subjects shifted their preference away from that choice key to the one that was correct for no-sample trials. Not surprisingly, there was an adverse effect of this training on performance in control food-sample trials, but this was only temporary and, by the end of training, performance had returned to its starting value. An analysis of the final two-session block indicated that there was a significant difference in the probability that the same choice stimulus would be selected on directed-forgetting and control food-sample trials $[F(1,7)=18.03, p<.01]$. Inspection of the data for individual subjects revealed that choice of the color appropriate to the food sample was below chance on the directed-forgetting trials in all subjects but one.

The results of the two transfer tests are shown in Figure 3. The percentage choice of the color or orientation corresponding to the food sample is plotted separately for food-sample trials with and without the clicker. On the control food-sample trials, choice responses were directed primarily to the choice key that was reinforced on food-sample trials. Although the graphics suggest that selection of the correct orientation choice key occurred more frequently than that of the correct colored choice key, statistical analysis indicated that the difference was not significant $[\mathrm{F}(1,7)=1.96, \mathrm{p}>.10]$. Presentation of the clicker after the food sample produced a substantial shift in choice responding towards the color or orientation that was correct for no-sample trials. Statistical analysis revealed that the difference in percentage choice of the food-related choice key on food-sample trials with and without the clicker was significant $[\mathrm{F}(1,7)=76.4$, $\mathrm{p}<.01]$. Examination of individual subject data revealed that seven of the eight subjects responded to the choice key appropriate to the food sample below chance on the directed-forgetting transfer trials $[F(1,7)=50.9, p<.01]$.

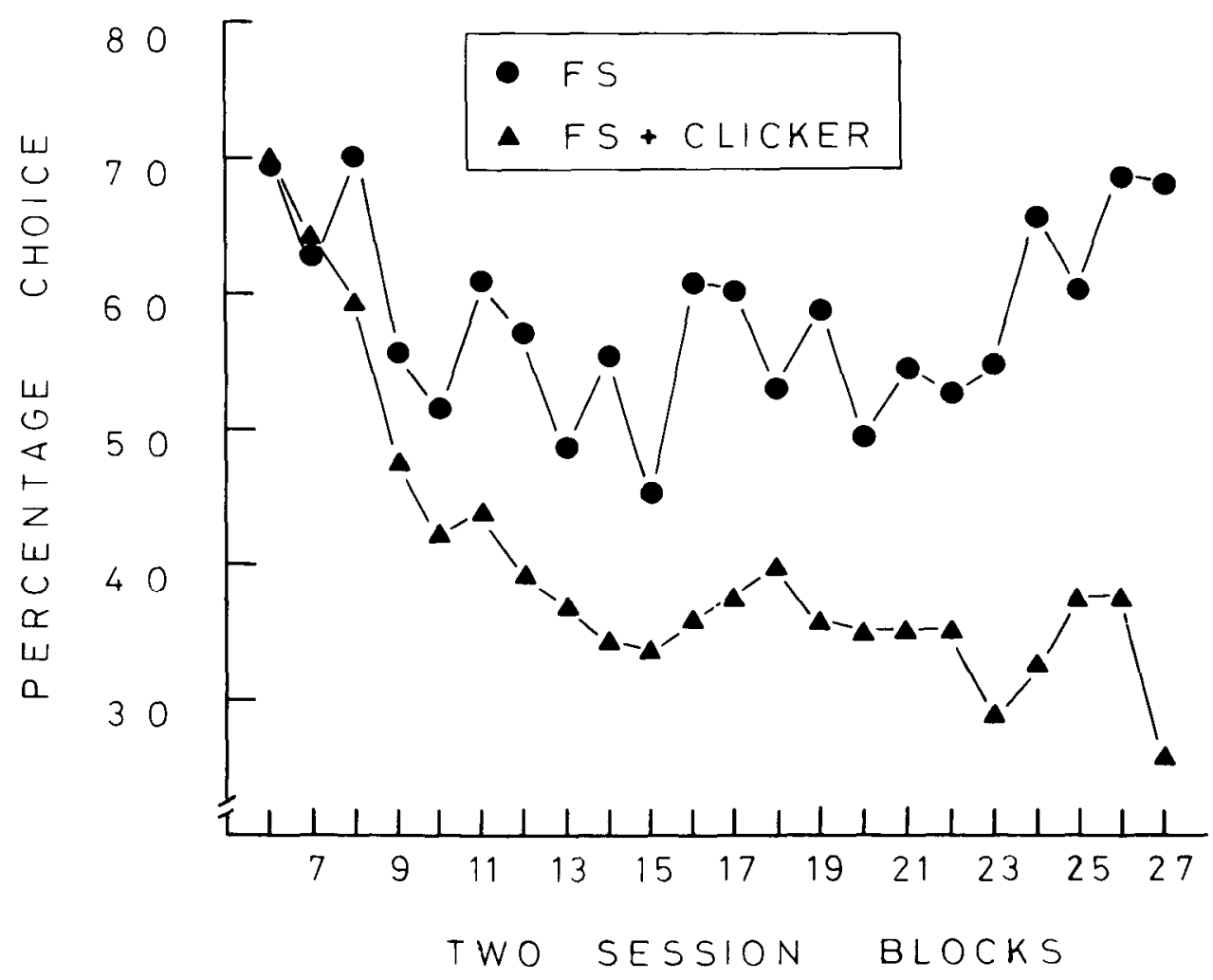

Figure 2. Percentage choice of the colored choice key corresponding to a food sample on standard food-sample DCD trials (FS, circles) and directed-forgetting trials (FS + Clicker, triangles). 


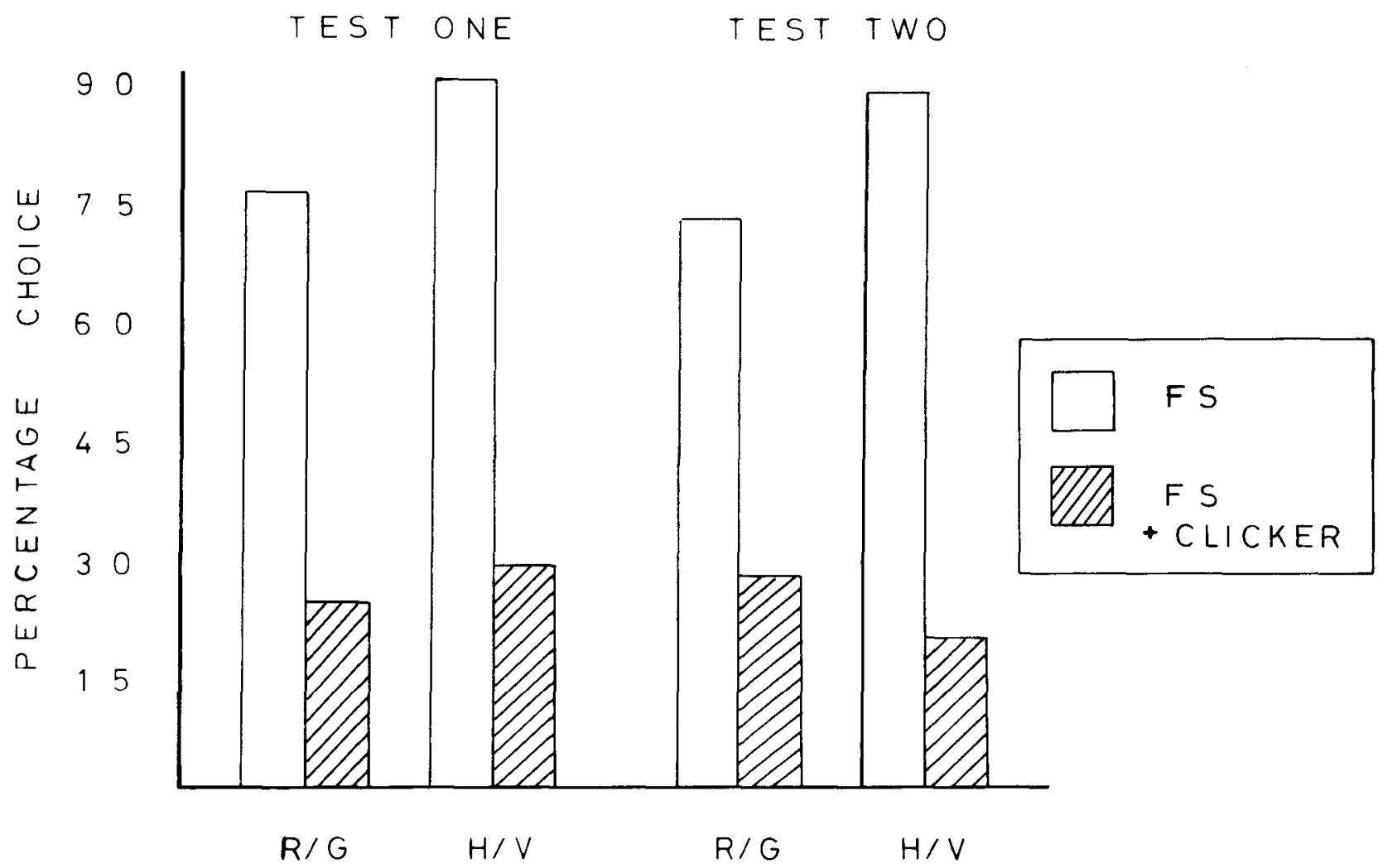

Figure 3. Percentage choice of the color $(R / G)$ or orientation $(H / V)$ choice key corresponding to the food sample on food-sample trials with the clicker (hatched bars) and without the clicker (plain white bars) is shown separately for the two transfer test sessions.

Performance on the no-sample trials remained at a highly accurate level throughout these two tests. The percentage correct selection was 89.1 and 81.3 on trials with orientation choice stimuli and 92.2 and 93.8 with the colored choice stimuli for the two tests, respectively. An analysis of these data with those for the standard food-sample trials revealed a significant interaction between the type of sample and the identity of the choice stimuli $[F(1,7)=8.37$, $p<.05]$. Subsequent analyses indicated that there was no difference in the percentage correct on food and nosample trials with orientations as choice stimuli $(F<1)$, but that performance was significantly better on no-sample trials than on food-sample trials with colors as choice stimuli $[F(1,7)=12.32, \mathrm{p}<.01]$. This pattern reflects that found at the end of directed-forgetting training.

\section{DISCUSSION}

This experiment examined a new method for establishing a cue as an instruction to terminate posttrial processing of information about an event. Pigeons were trained to select one of two choice stimuli if food had preceded their presentation. Choice of the other stimulus was reinforced if food had not been presented or if the food sample had been followed immediately by a clicker (cue to forget food sample). The finding that the clicker successfully con- trolled choice responding on trials with a transfer set of choice stimuli is consistent with the view that the clicker modulated posttrial processing of the food sample. Although the present design did not include a condition in which the effect of an auditory stimulus that had not undergone exposure to a directed-forgetting procedure was assessed on transfer trials, there are several reasons for supposing that the successful transfer was due to its training history. First, the fact that the clicker acquired control over choice responding only after extensive training and the fact that the initial introduction of the clicker did not produce this pattern eliminate any explanation of the transfer data in terms of some unconditioned effect of the clicker. Second, Colwill (1982) has shown that an auditory stimulus that is associatively neutral and familiar in a different context does not interfere with choice performance on food-sample DCD trials. Third, any nonspecific disruptive effect of the clicker should have reduced DCD performance only to chance level. However, selection of the choice key appropriate to the food sample occurred on fewer than $50 \%$ of the directed-forgetting transfer trials in all but one of the subjects. These data are, therefore, consistent with the idea that the cue to forget does, in fact, cancel the memory of the food sample. In the framework of controlled processing, it does so presumably by instructing the subject not to continue rehearsing the food sample. 
Before accepting the controlled processing account of these data, it is worth examining the applicability of other explanations that have been offered for the effect of a cue to forget that do not invoke the concept of controlled processing. One account appeals to an inhibitory process. The idea that the cue to forget is functionally identical to a conditioned inhibitor has been considered by Maki and his colleagues (see Maki, 1981) but found to be inadequate. However, although it seems unlikely that the cue to forget becomes an inhibitor of the memory trace of the food sample, it is possible that the training procedure used here establishes the cue to forget as an inhibitor of the choice response. The operations to which the subjects are exposed can be characterized as follows: (1) food sample + choice key = food; (2) food sample + choice key + clicker $=$ no food. If we use $A$ to denote the food sample + choice key combination and $\mathrm{X}$ to denote the clicker, we can describe the above operations in the form $\mathrm{A}+$ and $A X-$. This is precisely the procedure employed to establish $\mathrm{X}$ with inhibitory properties. Thus, as a consequence of this exposure, $X$ should become an inhibitor for the choice response. The problem with this account is that it fails to explain why subjects respond at all on the directed-forgetting trials. One would have to assume that this DCD task does not involve a conventional choice procedure. Rather, the subject solves the task asymmetrically. Thus, the pigeon learns to execute a default response, selection of one choice key, except when certain, very specific conditions hold. Then the alternative specific choice response is excited and executed. Hence, a case could be made that the directed-forgetting training does establish the clicker with inhibitory properties such that the arousal of the specific choice response is inhibited, leading to performance of the default response. It should be realized that this argument leaves unspecified the exact nature of the default response. Presumably, such a strategy involves some fairly sophisticated representation of the task and, in particular, the default response, since the ability of the clicker to inhibit the specific choice response and facilitate the default response transferred to a completely different set of choice stimuli. This in itself is evidence of a powerful cognitive process in the pigeon but not evidence of a controlled process.

Kendrick et al. (1981) have proposed a somewhat different account of the effect of a cue to forget. They suggest that the behavior occurring immediately prior to presentation of the choice stimuli serves as an important cue for retrieving information about the sample. Whether or not the cue to forget will produce a performance decrement will depend on the extent to which it disrupts this terminal behavior and hence retrieval of the sample. Applied to the present data, one would have to assume that the clicker elicits (presumably through a conditioning operation) terminal behavior that is identical to that exhibited on trials without a sample. Unfortunately, we do not have the necessary observational data that would permit evaluation of this account.
The above accounts share the assumption that, in one way or another, choice performance is based on a representation of the sample stimulus and that the cue to forget affects that representation. An alternative view that has recently been proposed is that subjects encode the sample in terms of the appropriate choice response (Honig, 1978; Roitblat, 1981). Thus, on food-sample trials, subjects remember to peck a particular choice stimulus, and on no-sample trials, they remember to respond to the other choice stimulus. The clicker could then activate the instructional representation associated with a no-sample trial. This version naturally anticipates the observed performance on transfer test trials. However, the main problem with this account is that I have uniformly failed to obtain evidence that the DCD task used here is, in fact, solved in this way (Colwill, 1982).

If these data are to be viewed as suggestive of the possibility that animals can, under some circumstances, disengage the rehearsal process, it should be done so cautiously. It should be emphasized that the sample of subjects that actually completed the experiment was very small. More than half of the animals, even after extensive training, showed no indication of solving the directedforgetting task and had to be discarded from the study. Consequently, the test data are derived from a group of pigeons that may not be entirely representative. The obvious difficulty that this task presented for the subjects may render it somewhat unsuitable for further analyses of controlled processing.

\section{REFERENCES}

Bsork, R. A. (1972). Theoretical implications of directed forgetting. In A. W. Melton \& E. Martin (Eds.), Coding processes in human memory. Washington: Winston.

BoWER, G. H., \& WINZENZ, D. (1970). Comparison of associative learning strategies. Psychonomic Science, 20, 119-120.

Colwill, R. M. (1982). Associative learning and post-trial processing. Unpublished doctoral dissertation, University of Cambridge.

Colwill, R. M., \& DickINson, A. (1980). Short-term retention of "surprising" events by pigeons. Quarterly Journal of Experimental Psychology, 32, 539-556.

Craik, F. I. M., \& Tulving, E. (1975). Depth of processing and the retention of words in episodic memory. Journal of Experimental Psychology: General, 104, 268-294.

Glenberg, A., Smith, S. M., \& Green, C. (1977). Type I rehearsal: Maintenance and more. Journal of Verbal Learning and Verbal Behavior, 16, 339-352.

Grant, D. S. (1981a). Short-term memory in the pigeon. In N. E. Spear \& R. R. Miller (Eds.), Information processing in animals: Memory mechanisms. Hillsdale, NJ: Erlbaum.

Grant, D. S. (1981b). Stimulus control of information processing in pigeon short-term memory. Learning and Motivation, 12, 19-39.

GRANT, D. S. (1982). Stimulus control of information processing in rat short-term memory. Journal of Experimental Psychology: Animal Behavior Processes, 8, 154-164.

HASHER, L., \& ZACKS, R. T. (1979). Automatic and effortful processes in memory. Journal of Experimental Psychology: General, 108, 356-388.

HonIG, W. K. (1978). Studies of working memory in the pigeon. In S. H. Hulse, H. Fowler, \& W. K. Honig (Eds.), Cognitive processes in animal behavior. Hillsdale, $\mathrm{NJ}$ : Erlbaum. 
Kendrick, D. F., Rilling, M., \& Stonebraker, T. B. (1981). Stimulus control of delayed matching in pigeons: Directed forgetting. Journal of Experimental Analysis of Behavior, 36, 241-251.

LABERGE, D. \& SAMUELS, S. J. (1974). Toward a theory of automatic information processing in reading. Cognitive Psychology, 6, 293-323.

MAKI, W. S. (1981). Directed forgetting in animals. In N. E. Spear \& R. R. Miller (Eds.), Information processing in animals: Memory mechanisms. Hillsdale, $\mathrm{NJ}$ : Erlbaum.

Posner, M. I., \& Snyder, C. R. R. (1975). Attention and cognitive control. In R. L. Solso (Ed.), Information processing and cognition: The Loyola Symposium. Hillsdale, NJ: Erlbaum.

RoItBlat, H. L. (1981). Codes and coding processes in pigeon shortterm memory. Animal Learning \& Behavior, 8, 341-351.

Rundus, D. J. (1971). Analysis of rehearsal processes in free recall. Journal of Experimental Psychology, 89, 63-77.
Shiffrin, R. M., \& Schneider, W. (1977). Controlled and automatic human information processing: II. Perceptual learning, automatic attending, and a general theory. Psychological Review, 84, 129-190.

Stonebraker, T. B., \& Rilling, M. (1981). Control of delayed matching-to-sample performance using directed forgetting techniques. Animal Learning \& Behavior, 9, 196-201.

Stonebraker, T. B., Rilling, M., \& Kendrick, D. F. (1981). Time dependent effects of double cuing in directed forgetting. Animal Learning \& Behavior, 9, 385-394.

(Manuscript received March 21, 1983; revision accepted for publication May 20, 1984.) 